\title{
A STUDY OF LITHOPONE
}

\author{
BY W. J. O'BRIEN
}

Lithopone is an intimate mixture of barium sulphate and zinc sulphide precipitated from a solution of barium sulphide and zinc sulphate. It is put through a special process by which its physical properties are changed so that it is made suitable for use as a pigment.

Lithopone is known as Griffith's White, Jersey Lily White, Beckton White, Ponolith, Oleum White, etc. These whites are practically 30 percent zinc sulphide and $7^{\circ}$ percent barium sulphate. Theoretically, equivalent solutions of barium sulphide and zinc sulphate produce 29.5 percent zinc sulphide and 70.5 percent barium sulphate. Some manufacturers in Germany reduce these mixtures with barytes and use seals which indicate the quality. As a general rule, green seal German or Dutch lithopone contains the maximum amount of zinc sulphide which is 29.5 percent. Next comes red seal, which varies from 24 to 26 percent; then blue seal, white seal, and yellow seal, which contain varying amounts of zinc sulphide according to the whim or custom of the manufacturer. There are, however, two grades of lithopone, one known as gold seal and another as bronze seal, which contain between 40 and 50 percent zinc sulphide, and naturally have much more hiding power and tinctorial strength than the so-called "green seal." These are made by adding an additional percentage of pure zinc sulphide to the pulp prior to the roasting operation, which is described later on. Sulphopone contains calcium sulphate instead of barium sulphate.

There has been a growing tendency during the past thirty years to replace white lead as a base for white pigments by zinc sulphide with other mixtures such as barium sulphate, magnesia, calcium sulphate, etc.

Ostwald" states that "there are certain difficulties with

${ }^{1}$ Zeit. Elektrochemie, 2, 946 (1905). 
the pale shades of color. The ground color of many pigments is white lead and white lead gradually becomes brown and dark. Further, colors, such as ultramarine, cadmium yellow, and vermilion, which contain sulphur, are acted upon by white lead and become dark. It is, therefore, desirable to substitute another white material for white lead. Lithopone can be used for this purpose."

Lithopone has many characteristics which make it a very valuable pigment. It is brilliant white, very fine in texture and according to Toch $^{1}$ has the same tinctorial strength but more hiding power than pure zinc oxide. It has also the advantage over white lead in that it can be mixed with pigments containing sulphur.

"Lithopone" is likewise very largely used in the cheaper grades of enamel paints, because it does not combine with rosin or semi-resin varnishes, and, therefore, remains unaltered in the package....

"As an inferior white, a first coat white, a ready mixed flat paint for surface, or as a pigment in the lighter shades for floor paints, lithopone cannot be excelled for its body, durability, hardness, fineness of grain, and ease of application. It does not oxidize progressively and this single feature has made it invaluable to the table oilcloth and floor oilcloth industry throughout the world... As a marine paint where other whites would be necessary, it is found to outlast both zinc oxide and lead carbonate."

It might be worth while to mention here the work of $\mathrm{M}$. Ragg $^{3}$ on the effect of lithopone on linseed oil. This author finds that neither linoleic acid nor linseed oil has any marked effect at the temperature of the water bath upon the hydrated zinc sulphide, zinc sulphide, or lithopone. At a higher temperature the hydrated zinc sulphide is acted upon to some degree by the acids of linseed oil, stearic acid, and oleic acids;

1 Toch: "Chemistry and Technology of Mixed Paints," 26.

2 Toch: Ibid., 30.

${ }^{3}$ Ragg: Farben Ztg., 15, No. 4I (1910). 
linseed oil does not act upon the hydrated zinc sulphide or upon lithopone.

In an address on zinc, lithopone, and lead, before the Ohio Association of Master House Painters and Decorators, Mr. H. A. Gardner says: "Preliminary long time exposure tests have shown that lithopone may become a valuable component of colored paints, even for exterior wooden surfaces. Up to the present time, however, a satisfactory exterior white lithopone paint for wood has not, to the writer's knowledge, been prepared. Nevertheless the fact that this pigment is, however, finding considerable usage in the making of paints for cement, is well known.

"Lithopone is today finding a wider application than any other pigment in the decoration of interior wall surfaces. Nearly all of the modern flat wall paints contain a very large percentage of this opaque white pigment often in combination with zinc oxide. The varnish vehicles used with these pigments in flat wall paints are especially suited for inside wall work, the completed mixtures having proved much more satisfactory than the old-style paste pigments ground in oil and subsequently washed with turpentine. The ease of application and the great extent of surface covered by one or two coats of lithopone-zinc flat wall paints have created a wide demand for painted surfaces... Actual tests made by the writer have shown that a properly prepared zinc-lithopone flat wall paint has a spreading power forty percent greater than the old-style washed pigment-oil pastes. These tests, moreover, demonstrated the superior hiding power of lithopone paints as well as their greater ease of working. The evident economies of their use are so overwhelming that it is safe to predict that for interior work they will be universally used within a comparatively short period of time, and, further, that the custom of painting the interior walls of all kinds of structures will grow to such an extent that the painter will be a more important factor in our building trades than ever before." 
According to the Farben Zeitung, ${ }^{1}$ lithopone has the following advantages:

I. It is free from poisonous properties and has greater covering properties than white lead.

2. It has great covering properties and high tinctorial strength.

3. It is unaffected by sulphur gases and mixes easily with oil and other colors.

"The following is a comparison of the covering properties of the following pigments:

22 parts Green Seal Lithopone are equivalent to

29 parts Zinc White

49 parts White Lead

50 parts Blanc Fixe

In addition, lithopone is cheaper than white lead."

According to a private communication from Mr. Maximilian Toch these comparative data are not accurate. $\mathrm{He}$ finds that twenty parts of green seal zinc lithopone are equivalent to twenty parts of white lead, thirty parts of chemically pure zinc oxide, and one hundred fifty parts of blanc fixe. This is for hiding power and not for covering capacity.

The earliest reference which has been found for the production of lithopone is in the English patent of John B. Orr, February 10, 1874. This patent according to Hurst ${ }^{2}$ is as follows: "This process consists in first preparing barium sulphide by calcining barytes with charcoal for some hours at a white heat; the calcined mass is then lixiviated with water to dissolve out the barium sulphide which is formed. The solution so obtained is divided into two equal portions. To one of these is added a solution of zinc chloride, whereby a precipitate of zinc sulphide is obtained according to the equation

$$
\mathrm{BaS}+\mathrm{ZnCl}_{2}=\mathrm{BaCl}_{2}+\mathrm{ZnS} .
$$

"The precipitate is not separated, but to the mass is now added the rest of the barium sulphide and sufficient solution

${ }^{1}$ Farben $Z$ tg., 6, 275 (1901).

2Hurst: "Painters' Colors, Oils and Varnishes," 66. 
of zinc sulphate, when a combined precipitate of zinc sulphide and barium sulphate will be obtained. This precipitate is washed with water, filter-pressed, dried, and then calcined at a red heat in a suitable furnace. While still hot the mass is thrown into cold water, which causes it to become rather denser than it otherwise would be, and thus have more body; the product is ground as fine as possible and dried, when it is ready for use."

Dr. S. Riderer ${ }^{1}$ described the process also. He states that solutions of barium sulphide and zinc sulphate are mixed in molecular proportions, the resulting precipitate of zinc sulphide and barium sulphate is then dried, heated in a furnace, ground, washed and reduced.

"The barium sulphide is obtained by roasting ground barium sulphate with coal or other carbonaceous material in a reducing atmosphere. Two types of furnaces are used; iiz., flat-bed reverberatory and rotary kiln (Bruckner), either is equally good. Lixiviation of the product of the roasting gives a solution of sufficient purity for the next stage of the process."

"For the zinc sulphate solution, any zinc-bearing material may be used. Spelter and dross leave little or no zinc in the insoluble residue but zinc ashes usually leave so much mud in the solution tanks that the unit of zinc in the material is not so valuable. As regards impurities, the sulphide metals cause little trouble. Arsenic or antimony ustually disappear as arsine or stibine where metallic zinc is used or are precipitated with copper and cadmium by adding some barium sulphide solution. Iron and manganese are usually removed by boiling with bleaching powder. A more powerful oxidizing agent and longer boiling are usually necessary when manganese is present."

There are a number of facts in the manufacture of lithopone which are of interest. Sowies ${ }^{2}$ points out that the presence of natural barytes in lithopone has a harmful effect.

The barium sulphate can only be obtained in a suitable

${ }^{1}$ Jour. Soc. Chem. Ind., 28, 403 (1909).

2 Sowies: Farben Ztg., I8, III7-9 (19I3). 
condition when it is precipitated with the zinc sulphide. In this way an intimate mixture is obtained, the barium sulphate adsorbing probably the zine sulphide.

Heating the barium sulphate-zinc sulphide precipitate affects its physical condition in a number of ways. In the first place, the zinc sulphide as precipitated from solution is highly hydrated. The composition of the precipitated zinc sulphide according to A. Souchay ${ }^{1}$ is as follows:

When dried

at room temperature at $100^{\circ}$ at $150^{\circ}$

Heating dehydrates the zinc sulphide. Hydrated zinc sulphide tends to work smeary on grinding. The effect of heat on the zinc sulphide is very similar to that obtained by Farnau ${ }^{2}$ on chilling the zinc sulphide to a very low temperature. The zinc sulphide becomes brittle and capable of being crushed to a very fine powder. A denser substance is also obtained, thereby increasing the body of the pigment.

The great danger in heating the zinc sulphide is that the zinc sulphide is easily oxidized to zinc oxide. A slight oxidation cannot be avoided even in a most favorable atmosphere. This oxidation not only decreases the yield of the pigment but hurts the body of the pigment as well. According to a recent paragraph, Zerr ${ }^{3}$ says:

"Lithopone manufacturers decided that by technically pure lithopone is understood one that contains besides the barium sulphate and zinc sulphide not more than one percent zinc oxide." A higher content of zinc oxide is to be objected to since it is shown by experiment that the higher the zinc oxide content the less stable is the lithopone to weather conditions.

1 Zeit anal. Chem., 7, 78 (1868).

${ }^{2}$ Jour. Phys. Chem., I7, 639 (1903).

${ }^{3}$ Farben Ztg., 17, I381 (1912). 
W. Hurschell ${ }^{1}$ says that " the ground material barium sulphate cannot be substituted for the precipitated barium sulphate since it has other properties. On the other hand, the addition of zinc oxide helps to prevent the yellowing of the oil in dark moist rooms."

It is doubtless true that zinc oxide hurts the body of the pigments somewhat, but of all additions to prevent darkening it is the least harmful and adds other good qualities to the lithopone. In a communication to us Mr. H. A. Gardner states that the addition of zinc oxide and whiting improves the weathering qualities of lithopone. Mr. Gardner's experiments were made on two-foot boards and have never been tried out on a large scale. We have been told by others that up to now lithopone is not suitable for exterior paint purposes, even when admixed with other materials.

There is also a tendency for an overheated pigment to have a yellowish tinge. This yellow color is due to the zinc oxide film as was shown by Farnau."

"These observations shed light on another set of phenomena, the change of color of oxides when hot and cold. When zinc oxide is heated in a matrass in the Bunsen flame, it becomes yellow; but returns to its original color when cold. Bismuth oxide and stannic oxide similarly treated, become darker when hot and lighter in color when brought back to room temperature, but do not become white again. Cooling the heated oxides causes disintegration of the coalesced particles and in their original state of fine subdivision, the masses show more or less their original color. This disintegration is not complete in the case of bismuth and stannic oxide.

"An experiment was made by heating the surface of zinc oxide contained in a crucible with the point of an oxyhydrogen flame. A good deal of volatilization occurred. On cooling, the mass of material regained its original color, but that which

1 Farben Ztg., I7, I 592 (I9I2).

${ }^{2}$ Farnau: Jour. Phys. Chem., I7, 639 (I9I3). 
sintered together by immediate contact with the flame remained yellow. It was still yellow after several months."

The only apparent reason for quenching in water is to prevent too much oxidation. Red-hot zinc sulphide exposed to the air oxidizes very readily. It was found by us that hot zinc sulphide does not oxidize in an atmosphere of carbon dioxide.

Lithopone has one peculiar property, that of turning gray in the sunlight and becoming white again in the dark. Toch says:" "Under normal circumstances if lithopone be mixed with any vehicle containing linseed oil or varnish and exposed to the sunlight, it turns gray very readily, and if again placed in the dark it returns to its normal white color."

There is frequent mention in the literature of this strange phenomenon and although the darkening of lithopone was observed at least thirty years ago, no satisfactory explanation of it has been offered up to the present. As Ostwald says: "It is still a riddle."

The earliest work done on the darkening of lithopone is that of Dr. Phipson. ${ }^{2}$ Phipson made a number of observations but could advance no satisfactory explanation for the facts observed. Dr. Phipson observed that a gate post painted white with lithopone turned black in the sunlight and became white again in the dark.

"I found that when this white powder [lithopone] was spread upon a piece of paper and exposed to the direct rays of the sun, it became fawn colored, brown, and finally a dark slate color in the course of about twenty minutes. When in this state it was placed in darkness, it became white again in two and a half to three hours time."

He first thought the color was due to barium sulphide. "The phenomenon was doubtless due to the use of sulphide of barium in the preparation of the pigment; for specimens made by another process in which no sulphide of barium was

1 Toch: "Chemistry and Technology of Mixed Paints," 28.

2 Phipson: Chem. News, 43, 283;44, 83 (I 88 I). 
used, did not show it at all." It then occurred to him that it might be silver.

"Of course these phenomena could not be due to any compounds of silver; nevertheless specimens were tested for silver, and with great care, but without the slightest result."

Finally he suggested the existence of a new element which he called actinium.

"It was hinted that I was dealing with a new element (to be called actinium) but continuation of my experiments led me to believe that the phenomenon described above may be probably due to the presence of sulphide of barium and protoxide of iron in the specimens rather than to some unknown metal.

"I observed that the dark slate-colored product was immediately soluble in cold acetic acid (as sulphide of iron is); and if the dark color be due to the production of sulphides of iron, the return of the pigment to its original white tint when placed in darkness for some hours, may be owing to the gradual oxidation of this sulphide of iron and consequent disappearance of the dark color.

"I found also that a strong calcination with free access of air, destroys, or considerably hinders the production of the actinic phenomena. I have also observed that although this pigment will retain this property of darkening in the sunlight for more than a year, some specimens have lost this property in a few months; and the gate post painted white after darkening, in the manner above mentioned, for the space of several days, will at last remain white."

In another article Phipson ${ }^{1}$ draws the following conclusions:

I. The phenomenon the [blackening] is due to a reduction which when the substance is removed to the dark becomes an oxidation and the pigment is restored to its original whiteness.

2. Pyrogallic acid facilitates the reduction.

3. The dark product is soluble in acetic acid, which allows it to be separated from the zinc sulphide.

1 Phipson: Chem. News, 44, 73 (I88I). 
4. The original white pigment allowed to remain for two nights and days in a large excess of acetic acid does not, previous to the action of light, dissolve the substance which is affected by this action (and this points to sulphide of zinc itself as being the substance affected).

"The constant minute quantities of iron in the numerous samples of these white pigments which I have examined led me to believe that the dark slate-colored product obtained by exposure to sunlight was owing to the production of the sulphide of iron, or to some compound sulphide of iron, zinc, and barium as mentioned in my first note. In order to decide this point, if possible, I took a specimen of the pigment which contained very little iron, and by exposing it over and over again to the alternate action of sunlight (reduction) and the darkness (oxidation) - renewing the surface after each experiment-I hoped to oxidize the iron completely, so that a moment would arrive when the pigment would no longer darken on exposure to the light. But, although the experiment was continued on a very thin layer, the newly exposed surface continued to darken as at first. It appears probable that the minute quantities of iron present are not the cause of the phenomenon."

Mr. Cawley also made many observations on the darkening of lithopone which appear in three papers, ${ }^{1}$ two in I88I and the third, ${ }^{2}$ ten years later. The writer did not discover this paper, hidden under the title, "The Curious Behavior of Certain Zinc Compounds," until after we had duplicated many of Mr. Cawley's results independently. These results will not be given with Mr. Cawley's since they will be discussed more in detail later.

While Mr. Cawley made many observations, he could advance no satisfactory explanation for the facts observed. Mr. Cawley studied the darkening of lithopone and has the following to say about it:

1 Chem. News, 44, 51, I67 (I88I).

${ }^{2}$ Ibid., 63, 88 (1 $89 \mathrm{r}$ ). 
"In general, however, it [lithopone] is subject to the grave defect of darkening when exposed to the sunlight, the darkening apparently being due not only to light but also dependent on the conditions of the atmosphere in respect to moisture content, etc. I have prepared pigments so sensitive as to be turned almost black when exposed to the sunlight for one or two minutes. A curious thing to be noted in this connection is that the darkened pigment will completely regain its whiteness when placed in the dark for a few hours, and when so bleached it appears to be somewhat less sensitive than it was originally. It would be a long story to go into all the details of experiments made by me in connection with this matter. I will, therefore, content myself by giving a brief account of the more important observations, interpolating here and there remarks of an explanatory character.

"I. The dried hydrated zinc sulphide before ignition is not altered by exposure to light, after ignition it darkens readily.

"2. The white stand purest zinc blend obtainable was levigated and exposed to the light. No darkening was observed but the same blend, calcined so as to be slightly oxidized, was readily darkened.

"3. Zinc sulphide was prepared in a very finely ground condition and free from hydration by burning zinc in an atmosphere of sulphur vapor and collecting the product in a chamber, the temperature of which was high enough to prevent the condensation of the sulphur. The sulphide was unchanged by light but like the blend darkened readily after slight calcination. In connection with the observations above named, I may say, I had been struck by the resemblance which the color developed in the pigment by light bore to that of finely divided metal deposited from certain metallic solutions. I found, too, as might have been expected, that in the heating of hydrated zinc compounds, when air is excluded, oxidized zinc compounds are always formed, and in this way the idea was suggested to me that light might be able, under certain conditions, to effect the reactions which 
it is well known heat does. These reactions may be expressed by the equations

$$
\begin{aligned}
& \mathrm{ZnS}+2 \mathrm{ZnO}=3 \mathrm{Zn}+\mathrm{SO}_{2} \\
& \mathrm{ZnS}+\mathrm{ZnSO}_{4}=2 \mathrm{Zn}+2 \mathrm{SO}_{2} .
\end{aligned}
$$

According to this hypothesis the darkening is due to the formation of a film of metallic zinc, which, owing to its finely divided condition would readily oxidize in the absence of light; the bleached pigment would therefore be less sensitive than it was originally, as it would be protected somewhat by a film of non-sensitive zinc oxide. This theory explained easily all the observed facts for a time but was afterwards found to be untenable.

"4. Noting that the pigment moistened with water was more sensitive than when in a dry condition, a sample was dried at $120^{\circ} \mathrm{C}$ and while warm was placed in a glass tube which was sealed before the blow pipe. Result - no discolorization after one year's exposure.

"An attempt was made to apply this observation by thoroughly drying the pigment immediately before grinding in linseed oil, the latter being as free from water as possible, the idea being that the oil would exercise a protective effect similar to that of a sealed tube. Result-not satisfactory.

"5. Taking a sample of pigment not very sensitive, per se, it was noted that its sensitiveness was greatly increased by moistening it with a weak solution of zine sulphate, from which it was inferred that the sensitiveness might be due to imperfect washing. The most thorough washing, however, would not remove the basic sulphate formed in the process of ignition. Attempts were made to decompose this basic sulphate into zinc oxide and an inert sulphate by treating the pigment with solutions of the hydrates of potassium, barium, sodium, and calcium; the first three were rejected as they appeared to act on the zinc sulphide itself. Lime water exercised for a short time a decidedly protective effect. Afterward it seemed to lose its power of making the pigment permanent to light.

"The best results were obtained by mixing with the zinc 
sulphide about one percent of freshly precipitated magnesium hydroxide. The resulting pigment was fairly resistant to light but the presence of magnesium somewhat impaired its general qualities."

This is the extent of the research done on this problem that can be found in the literature. There are a good many patents published; the principal ones will be discussed later in the paper. The following is the experimental part done by us.

\section{Experimental Part}

The conditions most satisfactory for the blackening of the lithopone were first studied.

It was known that certain salts such as sodium chloride, zinc sulphate, etc., accelerated the blackening. Accordingly mixtures were made of a sample of lithopone that would darken with varying amounts of the different salts, ground with the same quantity of water. These were exposed on microscopic slides first to the action of the sun rays.

Lithopone plus

\begin{tabular}{|c|c|c|c|c|c|c|}
\hline & \multicolumn{6}{|c|}{ Percent } \\
\hline $\mathrm{XaCl}$ & I & IO & 20 & 30 & 40 & 50 \\
\hline $\mathrm{ZnSO}_{4}$ & I & IO & 20 & 30 & 40 & $5^{\circ}$ \\
\hline $\mathrm{ZnCl}_{2}$ & I & IO & 20 & 30 & 40 & 50 \\
\hline $\mathrm{CdSO}_{4}$ & I & IO & 20 & 30 & 40 & 50 \\
\hline
\end{tabular}

All the above salts accelerated the blackening. While the final intensity of darkening was the same in each case, the rate of darkening was different and there was an optimum percent of salt for the rate of darkening in each case. For example, it was found, keeping all the conditions the same, that a mixture of thirty percent zinc sulphate with seventy percent lithopone darkened more quickly than ten percent zinc sulphate with ninety percent lithopone.

The effect of acid and alkali on the rate of darkening was next observed. A mixture of thirty percent of zinc sulphate and seventy percent of lithopone was made into a thin paste 
as before and a little sulphuric acid added drop-wise to a very faint acidity. In the same way sodium hydroxide was added drop-wise to a portion of the same mixture till faintly alkaline. Portions of the two samples thus prepared were then rubbed on the glass slides and exposed. It was found that acid or alkali rather hindered the darkening, and that the darkening took place best in a neutral medium. Finally it was found that all salts which formed soluble zinc salts accelerated the darkening; while salts which formed insoluble zinc salts, such as the alkali phosphates, ferrocyanides, borates, cyanides, or bicarbonates stopped or retarded greatly the darkening. A discussion of these results will be taken up later.

The effect of moisture on the darkening of the lithopone was next studied. The results obtained agreed with Mr. Cawley's results already given. A piece of paper, covered with a moist suspension of lithopone was put into a quartz glass flask, which was then evacuated, sealed, and exposed to the rays of a large Macbeth printing lamp. The blackening took place more quickly than in air. This is probably due to the fact that moisture does not escape when the flask is sealed while it tends to evaporate in the air, the presence of moisture greatly aiding the darkening. On the other hand, a piece of paper prepared as above but dried in an air bath was exposed in a dry, evacuated quartz flask. No change was observed. This proves that moisture is necessary for the darkening.

It was also found that the lithopone darkened more readily in a moist hydrogen atmosphere or any atmosphere free from oxygen. The blackened lithopone did not become white again on being put into a flask which was then evacuated.

The reason for this is that the change from the black to the white color is evidently an oxidation and not a reversible reaction. Blackened samples of lithopone on paper were treated with a dilute solution of hydrogen peroxide. The blackened surface whitened immediately. The same effect was obtained when strips of paper, covered with lithopone and darkened, were held in an atmosphere of ozone. 
The ideal conditions found for making the lithopone most sensitive were an addition of thirty percent zinc sulphate to the lithopone which was then made up to a thin paste and exposed in an atmosphere of moist hydrogen. The atmosphere of moist hydrogen was secured by using a large test tube of Uviol glass and closing it with a two-hole rubber stopper through which the moist hydrogen gas was passed by means of a glass tube reaching up into the inverted tube. The thin paste of lithopone and zinc sulphate was applied to the glass slide by means of a spatula and rubbed to form a thin film. The glass slide was then put into the Uviol tube and partly exposed. The nature of the surface had also an effect on the reaction. It was found that the reaction went faster on roughened glass than on paper, wood, or smooth glass.

The source of light in all these experiments was a Macbeth printing lamp. This lamp is run on a I ro-volt circuit and requires about 25 amperes. The carbon rods are cored and filled with a special composition which gives, on burning, a very powerful light rich in the shorter wave lengths. The light is quite steady, the current being automatically controlled by means of a coil and plunger which regulates the distance between the burning ends of the carbon rods. A reflector of aluminum throws the light forward in one direction. This light is more satisfactory for most purposes than sunshine.

Our first thought was that the black product was iron sulphide. Hydrogen sulphide, formed by the decomposition of the zinc sulphide by light and moisture according to the reaction

$$
\mathrm{ZnS}+\mathrm{H}_{2} \mathrm{O}+\text { light }=\mathrm{ZnO}+\mathrm{H}_{2} \mathrm{~S},
$$

might react with iron salts in the lithopone forming the black ferrous sulphide. The ferrous sulphide in a very thin film would be oxidized to the light yellow ferric salt, accounting for the disappearance of the black color.

Lithopone made from chemically pure reagents was analyzed for iron by the colorimetric sulphocyanate method. Result--only a slight trace was found, that is, less than o.oor percent. This lithopone darkened when exposed to light. 
The black film could be removed by dipping the blackened lithopone carefully into water; the black film would float usually on top of the water, leaving a new surface beneath, which darkened as before. This could be repeated as often as desired. We concluded that it was unreasonable to believe that a mere trace of iron could cause all this darkening. This confirms Dr. Phipson's and Mr. Cawley's work.

Phipson" says that "the darkening was not due to iron as he first supposed or to other impurities such as one would most likely find in zinc salts such as cadmium, lead, arsenic, silver, bismuth, etc." John B. Orr ${ }^{2}$ states that if the pigment is properly made it does not contain iron, lead, arsenic, or manganese, and yet insolation produces this bluish coloration unless means be taken to prevent it. Having excluded the possibility of ferrous sulphide, the next step in the experimental work was to find out what the black product was. We could form no theory to fit in with the facts observed until we had definitely decided on what the black product was.

This problem offered many difficulties. One trouble in testing the black film was due to its extreme sensibility to reagents. It oxidized very readily in the air when isolated in any quantity. It also disappeared very quickly in water. It was very soluble in acetic acid and soluble in most salt solutions such as sodium chloride, sodium sulphate. It was quite stable in carbon bisulphide. This behavior could be explained by the fact that we were dealing with metallic zinc in a very fine state of division.

Microscopic tests were first tried. The fact that other zinc salts were present besides the metallic zinc eliminated the ordinary microscopic tests. Attempts were first made to spread a film of gelatine over the blackened surface and to allow a dilute solution of hydrochloric acid to diffuse in. The acid would act slowly on the zinc sulphide as well as the

${ }^{1}$ Phipson: Chem. News, 44, 73 (1881).

2 Orr: Ibid., 44, I2 (1881). 
metallic zinc if present, causing a slow evolution of hydrogen sulphide and hydrogen gas. Hydrogen sulphide is to a great extent soluble in gelatine whereas hydrogen is not and could be observed, if present, as minute bubbles of gas under the microscope.

Glass slides were, therefore, covered with a sensitive sample of lithopone and blackened quickly in a moist atmosphere of hydrogen, in this way avoiding as much as possible an oxide film on the blackened product. The slide with the blackened product and one unblackened were then covered with a film of gelatine. A large drop of dilute acid was placed on each slide and allowed to diffuse in. The test was not satisfactory, the gelatine did not adsorb entirely the hydrogen sulphide evolved and there were always observed on the blank some bubbles of gas, probably due to carbonates. On the other hand, there was a greater evolution of gas from the blackened product.

Electrolytic methods were then tried in the hope that if metallic zinc were present, metallic lead or copper would be thrown out of the solutions of these salts. It is well known that when metallic lead is thrown out of solution, it tends to form characteristic lead trees. Solutions of lead and copper of varying concentrations were, therefore, tried, and while the black product immediately dissolved in the solution of these salts, no trace of lead trees or metallic copper could be identified under the microscope. This could be accounted for by the fact that the black film is very thin and contains but a small amount of zinc, not sufficient in quantity to throw out the copper or lead.

There are a large number of reactions that show a color change in the presence of a reducing agent. A dilute solution of iodine colored blue with starch was found to be unsatisfactory as also was alkaline permanganate. Potassium ferricyanide was then tried. As is well known, potassium ferricyanide in the presence of a ferrous salt gives Prussian blue. This reaction is very sensitive.

The solutions used were about a ro percent solution of 
ferricyanide, ro percent solution of ammonium acetate and about o. I percent solution of ferric alum.

The method of testing for the zinc was as follows: A drop of the ferric alum solution and a drop of the potassium ferricyanide solution were placed side by side near the object to be tested for and then caused to run together. Where the ferric alum, the reducing substance, and the potassium ferricyanide solutions meet, a reduction of the ferric alum takes place and a blue color results.

Trials were first made on mere traces of zinc, such as light lines of zinc made by rubbing a piece of metallic zinc over some barium sulphate paper. With traces of zinc as small as these, the test worked beautifully. On trying it out on the blackened lithopone, however, the zinc sulphide present interfered, the hydrogen sulphide caused by the action on the zinc sulphide of the free acid present in the ferric alum, due to hydrolysis, also reduced the ferric alum. This was overcome to a great extent by adding ammonium acetate to the ferric alum, a large part of the free acid of the ferric alum being neutralized.

By adjusting carefully the amount of ammonium acetate added to the ferric alum, comparable results could be obtained.

In conducting the tests, one glass slide was covered with a sample of lithopone which was very sensitive to light. It was blackened by exposure to light. Another slide was covered with an undarkened sample of the same lithopone and then covered with a thin film of zinc dust, made by buffing a piece of metallic zinc on a carborundum wheel and catching the fine zinc dust on the slide covered with moist lithopone. In this way a very sensitive zinc dust could be obtained and one less liable to be covered with an oxide film. Another slide was taken and covered with the lithopone alone. The three slides, side by side, were tested as described above. The slide, covered with fine metallic zinc, acted instantly, as did the black product, giving the blue color, while the blue color appeared on the lithopone slide only after several minutes. While these tests do not prove that we have metallic zinc, 
since an unknown subsulphide of zinc may be present, all the facts point clearly to the formation of a film of very finely divided metallic zinc. The substance oxidizes readily, is extremely soluble in acetic acid, dissolves in solutions of sodium chloride, or sodium sulphate, is extremely soluble in alkalies, and is not soluble in carbon bisulphide.

That it is a direct product of the action of light on the zinc sulphide is shown by the fact that if the lithopone is shaken in acetic acid for some time and then washed and exposed, the blackening takes place as before, the black product, however, is quickly dissolved in acetic acid, showing that the action of light gave rise to a product soluble in acetic acid.

The reaction was studied under the microscope. A slide was covered with lithopone and exposed while under the microscope to the light of the arc lamp by directing a beam of light from the arc through a small opening in an asbestos board onto the slide. It was observed that the darkening took place in spots. A bubble of gas formed and then on the side of the bubble a black spot would appear, growing larger.

Cadmium sulphate acted in two ways when mixed with lithopone. In the air it accelerated the blackening; in a hydrogen atmosphere the blackening was also accelerated but some cadmium sulphide was also formed, the black showing a yellow tinge. Cadmium sulphide oxidizes very readily in the air and is probably oxidized as quickly as it is formed. In hydrogen this is not the case.

It was thought that perhaps cadmium sulphate hydrolyzed slightly, the free acid acting on the zinc sulphide setting free hydrogen sulphide. This was found not to be the case, a paper saturated with a solution of cadmium sulphate, placed near the exposed slide covered with lithopone, but not in direct contact with it, turned yellow, showing that the zinc sulphide in the presence of moisture in a hydrogen atmosphere is decomposed, one of the products being hydrogen sulphide. This is analogous to the action of magnesium sulphide on water.

$$
\mathrm{MgS}+2 \mathrm{H}_{2} \mathrm{O}=\mathrm{Mg}(\mathrm{OH})_{2}+\mathrm{H}_{2} \mathrm{~S} \text {. }
$$


The finer the lithopone is, the more easily should it be reduced, as there would be a greater surface exposed. That this is so was shown to be the case. Three samples of lithopone, one that was quite sensitive to light, one only slightly so, and one not at all, were found to show, under the microscope, appreciable difference in the size of the particles. The one most sensitive to light was found to be the finest grained. Measurements of the size of the particles under the microscope could only be approximate since the average size was found to be less than 0.0 I of a micron in diameter, a value too small for the ordinary microscope to handle accurately.

The sensitiveness of the zinc sulphide to light was thought to be due to the fact that it was carried down in a very fine condition by barium sulphate. Barium sulphate and zinc sulphide were precipitated in molecular proportions according to the equation

$$
\mathrm{BaS}+\mathrm{ZnSO}_{4}=\mathrm{BaSO}_{4}+\mathrm{ZnS} .
$$

The barium sulphate-zinc sulphide precipitate was filtered, washed, and dried at about $100^{\circ} \mathrm{C}$. This was then subjected to the following treatment: the barium sulphate-zinc sulphide precipitate was ground with zinc sulphate to a very fine powder, moistened, and exposed on glass slides to the light of the arc lamp. After three hours' exposure, no darkening was observed.

It was then thought that barium sulphate did not adsorb the zinc sulphide as efficiently as other substances, for example, aluminum hydroxide. Aluminum sulphate and zinc sulphate were mixed together and ammonium sulphide was added. A precipitate of zinc sulphide and aluminum hydroxide was obtained. This precipitate was washed, dried, and tested as the barium sulphate-zinc sulphide was, with no darkening taking place.

A colloidal solution of zinc sulphide was then prepared in the following manner: a zinc sulphate solution was poured into a solution of sodium hydroxide until the zinc hydroxide just barely dissolved. This was then diluted to about ten times its volume with water and a rapid stream of hydrogen 
sulphide passed through till the precipitate just dissolved. The colloidal zinc sulphide was then precipitated by adding aluminum chloride. In this way a very amorphous precipitate of zinc sulphide was obtained. On exposing this precipitate to the light with reducing agents and other sensitizing substances no darkening was obtained.

Chemically pure zinc sulphide was ground with barium sulphate in an agate mortar crusher. As we were unable to grind the zinc sulphide at ordinary temperatures, pieces of solid carbon dioxide were thrown into the agate mortar crusher, chilling the zinc sulphide and making it brittle. We were able to grind the zinc sulphide as fine as the sensitive lithopone. This zinc sulphide did not darken on exposure to light in presence of reducing agents.

Since we were unable to get zinc sulphide to darken by precipitating it in a finely divided condition, or by having it adsorbed, or by grinding it to a very fine condition, it was concluded that heat must be necessary for some unknown reason.

The following possible theories could be advanced as to the effect of heat on the barium sulphate-zinc sulphide. Since it is known that zinc sulphide melts at about $1440^{\circ} \mathrm{C}$ and in the presence of sodium chloride at a much lower temperature, it was thought probable that in the reducing atmosphere metallic zinc was formed which went into colloidal solution in the solid solution of zinc sulphide. The light acted as a pectonizing agent as did also the salts which accelerated the darkening, such as sodium chloride, zinc sulphate, etc. Another possible explanation offered itself in the possibility of a metastable compound being formed on heating which broke down under the action of light, depositing metallic zinc. There was still also the chance that some powerful reducing agent might be formed on heating the zinc sulphide-barium sulphate precipitate since it is known that reducing agents greatly aid the darkening of the lithopone. The presence of a powerful reducing agent in the lithopone seemed hardly probable since this reducing agent would be oxidized on standing in the air and moisture. 
The barium sulphate-zinc sulphide precipitate prepared as described in the previous work was heated in an electric tube furnace. An electric tube furnace was used because it was thought that atmospheric conditions could be better controlled and the temperature measured if desired. An objection to the tube furnace, however, was that only a small quantity could be heated at one time so relatively high percentage oxidation, as we found out afterwards, could not be prevented. This was due to the fact that the small quantity of hot zinc sulphide came in intimate contact with the air on pouring it from the tube into the water.

The tube furnace was evacuated and the barium sulphatezinc sulphide precipitate was heated for about half an hour at about $700^{\circ} \mathrm{C}$. It was then quenched in cold water, washed, dried, ground, and tested under the most favorable conditions for darkening. This product would not darken. This substance on analysis showed

\begin{tabular}{cc:c}
$\mathrm{BaSO}_{4}$ & $\mathrm{ZnO}$ & $\mathrm{ZnS}$ \\
\hdashline $7 \mathrm{I} .2 \mathrm{I}$ & 10.00 & 18.60
\end{tabular}

It was then thought that a certain temperature was most favorable for the production of a lithopone that would darken. The barium sulphate-zinc sulphide precipitate was then heated in the electric tube furnace which was kept well evacuated in order to prevent the oxidation of the zinc sulphide at a temperature of $500^{\circ} \mathrm{C}$. This product on testing showed no darkening. Another run was made at $800^{\circ} \mathrm{C}$ and a product was obtained which would not darken. Runs were made up to $1400^{\circ} \mathrm{C}$ under otherwise the same conditions with the idea that temperature played an important part in producing a lithopone that would darken. In no case was a lithopone obtained that would darken. Analyses of the substances obtained showed a high zinc oxide content.

Preventing excessive oxidation of the zinc sulphide on heating, is rather difficult. Zinc sulphide precipitated from 
an aqueous solution is always hydrated. A. Souchay's work on this has already been mentioned. This water is held in more or less intimate union with the zinc sulphide and it is possible that on heating it goes over to $\mathrm{ZnS} \times \mathrm{xZnO}$ as for example:

$$
2 \mathrm{Zn}(\mathrm{OH})(\mathrm{SH})+3 \mathrm{O}+\text { heat }=\mathrm{ZnS} . \mathrm{ZnO}+2 \mathrm{H}_{2} \mathrm{O}+\mathrm{SO}_{2}
$$

Oxidation of the zinc sulphide was prevented by mixing intimately in a mortar the barium sulphate-zinc sulphide with about five percent of common salt and about one percent of ammonium chloride and heating this mixture in a graphite crucible, covered tightly with a graphite cover. This mixture was heated in a muffle furnace at about $1000^{\circ} \mathrm{C}$ for about ten minutes. In this way a substance was obtained which was very sensitive to light.

The use of the ammonium chloride and the sodium chloride is to prevent oxidation. The ammonium chloride volatilizes at a low temperature and displaces most of the oxygen. The fused sodium chloride dissolves off the zinc oxide film that is formed about the zinc sulphide particles, giving a very white, fine sample of lithopone which, however, is very sensitive to light.

It was thought advisable to see if under the same conditions lithopone made as pure as possible would darken as much as lithopone made from commercial zinc sulphate and barium sulphide. Barium sulphide as pure as possible was obtained which contained only traces of impurities. It was dissolved in boiling hot water; the solution was allowed to stand until clear and then was poured into a solution of zinc sulphate which was also very pure. The precipitate was well washed, dried, and heated as described above. A product was obtained which darkened just as readily as the lithopone obtained from the impure barium sulphide and zinc sulphate.

Having found that chemically pure lithopone would darken it was next sought to find out whether barium sulphate was necessary for the darkening. Chemically pure zinc sulphide was made by precipitating zinc sulphide from a chemically 
pure solution of zinc sulphate. This precipitate was washed, dried, and ground to a fine powder. It was then ground with sodium chloride and heated as described above. The zinc sulphide so obtained darkened on exposure.

Having found an easy way of darkening the zinc sulphide alone, it was now sought to find out whether, on sudden cooling, a metastable compound was obtained as in the case of glass, which under the action of light broke down into metallic zinc.

By slow cooling it was thought that the formation of the metastable phase could be prevented and so a product that would not darken be obtained. Some zinc sulphide-barium sulphate precipitate was taken and heated to about $1000^{\circ} \mathrm{C}$ and then allowed to cool slowly. This product did not darken but analysis gave the following results

\begin{tabular}{c|cc}
\hline $\mathrm{BaSO}_{4}$ & $\mathrm{ZnO}$ & $\mathrm{ZnS}$ \\
74.06 & $\mathrm{I} 7.93$ & 8.64
\end{tabular}

To prevent excessive oxidation, another sample was run as before, but instead of allowing it to cool in air, it was cooled in an atmosphere of carbon dioxide. This product gave on analysis

\begin{tabular}{ccc}
\hline $\mathrm{BaSO}_{4}$ & $\mathrm{ZnO}$ & $\mathrm{ZnS}$ \\
\hline 69.49 & 2.76 & $27 \cdot 74$
\end{tabular}

and did darken.

The rate of cooling was now decreased with the idea that perhaps the cooling in the air was quick enough to carry the substance over to the metastable condition. Some more of the unheated barium sulphate-zinc sulphide precipitate was then heated in a quartz tube which was kept continually filled with carbon dioxide by means of a Kipp generator. The product was brought to a temperature of about $1000^{\circ} \mathrm{C}$ and then allowed to cool slowly for three hours. The product on analysis showed a low oxide content and darkened. 
The presence of some slightly soluble, though powerful reducing agent in the heated zinc sulphide-barium sulphate precipitate now occurred to us. On the face of it, this seemed improbable, as it is hard to see how a reducing agent could exist in lithopone without undergoing oxidation. On the other hand, it was shown that reducing agents greatly accelerate the darkening.

Tests were made for the presence of the reducing agent in the lithopone as follows: A very dilute solution of iodine was colored blue with starch. Zinc sulphide and barium sulphate were ground together in the proportions found in the lithopone that would darken. Uniform suspensions were then made by shaking equal weights of the barium sulphate-zinc sulphide and the lithopone in the same quantity of water and adding to each the same quantity of solution of iodine. Decolorization took place immediately with both samples.

A very dilute solution of alkaline permanganate was then tried; the lithopone seemed to act even more slowly on this solution than the barium sulphate-zinc sulphide, proving that there was no strong reducing agent formed by heating the lithopone.

Up to now we could obtain no satisfactory theory that would explain all the facts observed. Repeated analyses of many samples of lithopone which would darken and which would not, show a marked difference in their zinc oxide content. Below are some of the analyses of samples that would and would not darken.

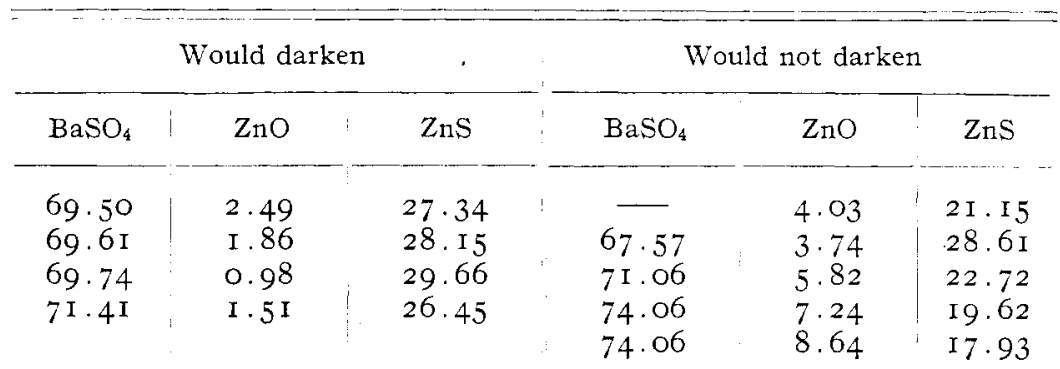

In no case did we get a sample that would darken, as made 
by us, whose oxide content ran much above 3 percent zinc oxide. The analyses were conducted as directed by Holley. ${ }^{1}$

It seemed evident then that we were dealing with a protective film of zinc oxide over the zinc sulphide, preventing its decomposition by light and moisture. If this were so, we should be able to remove the zinc oxide film from the zinc sulphide by boiling in an aqueous solution of zinc chloride, as it is known that zinc chloride dissolves zinc oxide. The zinc sulphide should then darken in the light even though it had not been heated.

Concentrated solutions of zinc chloride and sodium chloride were added to separate portions of chemically pure zinc sulphide in Erlenmeyer flasks, provided with return condensers and were boiled for some time. The zinc sulphide was originally slightly yellow but a beautiful white zinc sulphide resulted on boiling, showing that most of the zinc oxide film had dissolved. This zinc sulphide, well washed, was then mixed with a little sodium hydrosulphite and exposed in a moist hydrogen atmosphere to the light. It darkened readily.

We were unable to get zinc sulphide to darken without the use of a reducing agent. The reduction of the zinc sulphide in lithopone is probably helped greatly by the presence of barium sulphate which adsorbs zinc sulphide and the metallic zinc, since barium sulphate is known to adsorb gold. This was shown by Antony and Lucchesi, "Further, if barium sulphate and mercurous chloride are suspended in water, and excess of auric chloride then added, the barium sulphate takes up the gold and becomes the color of purple of Cassius."

Barium sulphate and zinc sulphide were precipitated together in the usual way, washed, dried, and ground. Exposure of the barium sulphate-zinc sulphide had no effect even with reducing agents. The barium sulphate-zinc sulphide was then boiled for a considerable time in concentrated zinc chloride solution. It was then washed a number of times by

1 Holley: "Analyses of Paint and Varnish Products," I 23.

2 Antony and Lucchesi: Jour. Chem. Soc., 72 II, 43 (1897). 
decantation and dried in air. The precipitate was then ground very fine and exposed. After some exposure, darkening occurred as we had expected, without the use of a reducing material. A solution of sodium chloride was found to work the same way as zinc chloride in dissolving off the zinc oxide film but not so energetically.

The presence of a zinc oxide film over the zinc sulphide explains the behavior of zinc sulphate, zine chloride, sodium chloride, and reducing agents in accelerating the darkening of the lithopone. They dissolve the oxide film away from the zinc sulphide as fast as it is formed, leaving always a sensitive zinc sulphide. The use of sodium chloride in heating the zinc sulphide in order to get a substance that will darken is also explained. Fused sodium chloride is a good solvent for zinc oxide.

This explanation is borne out by the many patents that have appeared in the literature on this subject. Rudolf Alberti, ${ }^{1}$ in a patent for making lithopone more stable, uses barium peroxide, hydrogen peroxide, and sodium peroxide. " $\mathrm{BaO}_{2} \cdot \mathrm{H}_{2} \mathrm{O}_{2}, \mathrm{Na}_{2} \mathrm{O}_{2}$ are used to oxidize the black material which blackens in the light. This material is converted to a higher oxide and is permanent to light."

The black material that Alberti has in mind does not exist. He is simply oxidizing the zinc sulphide, forming a protective layer over the zinc sulphide and so making it stable to light.

R. Steinau, ${ }^{2}$ in making a light and weatherproof lithopone, has the following to say: "The darkening of lithopone is due to the presence of zinc and cadmium chlorides. A lightproof lithopone can, therefore, be obtained if one uses a chlorine and oxygen adsorbing salt." He uses zinc carbonate or magnesium carbonate with alkali nitrite.

Mr. Steinau probably mixed some cadmium and zine chloride with lithopone and found that they accelerated the

\footnotetext{
${ }^{1}$ Alberti: Centralblatt, 1906, II, 65 I.

2Steinau: Ibid., I908, I, I 593.
} 
darkening, as we found. He, therefore, concludes that they are the cause of the darkening of lithopone. Just what he means by a chlorine or oxygen adsorbing salt is not quite clear. He probably means a reducing salt. One would hardly see the need of that as there is no free chlorine present. His patent is probably somewhat efficient because he gets an insoluble film of zinc carbonate formed. There is doubtless a small quantity of soluble zinc salts always held by the zinc sulphide-barium sulphate precipitate which in the presence of some soluble carbonate would form a film over the zinc sulphide.

Cawley ${ }^{1}$ makes lithopone fairly resistant to light by mixing one-half to one percent of freshly precipitated magnesium hydroxide. Mr. Cawley found that the presence of the magnesia somewhat impaired the qualities of the lithopone. The magnesium hydroxide forms a film over the zinc sulphide as before.

W. Ostwald ${ }^{2}$ claims that lithopone can be made stable to light if one washes the product with non-acid water solution of such salts as soda, potash, or alkali phosphates, borates, cyanides, bicarbonates, ferrocyanides, sulphides, sulphydrides, and hydroxides.

It is evident that Ostwald has picked out nearly all the soluble salts that form insoluble zinc salts. The significance of this is that an insoluble zinc salt is formed around the zinc sulphide which protects the zinc sulphide from the action of light. For example, he found alkali phosphates to work well. The slight traces of soluble zinc salts combined with the phosphate, forming zinc phosphate which, as we all know, is insoluble.

Allendorf ${ }^{3}$ does the same thing when he mixes with the lithopone, after heating, soap solutions of the earth metals or aluminum. In the United States patent $883, \mathrm{I}_{42}, \mathrm{P}$. Lihme oxidizes the zinc sulphide with some soluble nitrate.

${ }^{1}$ Cawley: Chem. News, 63, 88 (1891).

${ }^{2}$ Ostwald: Centralblatt, I908 II, 1707.

${ }^{3}$ Allendorf: Ibid., I909 I, I 16. 
"A soluble nitrate is added to the product heated in a muffle furnace, corresponding to the reacting or darkening products."

How he knows what the darkening products are and how much soluble nitrate to add is beyond the most lively imagination.

Bayer and Company, ${ }^{1}$ make lithopone stable to light by subjecting the finely divided lithopone, in suspension in the anode chamber in water containing sufficient electrolyte, to the electric current.

W. Brase? makes light-proof lithopone by adding to the moist or dry lithopone 0.5 percent potassium nitrate.

W. Ostwald ${ }^{3}$ claims to make lithopone stable to light by burning and cooling in absence of oxygen. The air is completely removed and an indifferent gas passed in.

Ostwald probably burns the hydrated zinc sulphide so that it oxidizes according to this equation

$$
2 \mathrm{Zn}(\mathrm{SH})(\mathrm{OH})+\text { heat }=\mathrm{ZnS}+\mathrm{ZnO}+\mathrm{H}_{2} \mathrm{O},
$$

the zinc oxide forming around the zinc sulphide.

There are a number of other patents which embody the same idea, an insoluble film over the zinc sulphide.

The objection to all these applications, for preventing the darkening of lithopone, is that they hurt the good qualities of the lithopone. The presence of an alkali salt is bound to cause trouble sooner or later with the oil. Alkalies also cause the lithopone to turn yellow when mixed with the oil.

To prevent the darkening of lithopone a coating over the zinc sulphide is necessary. This hurts the strength and covering properties of the lithopone, however, and a choice should be made of an additive substance that affects the quality of the lithopone the least and which adds some good qualities. In this laboratory we were able to obtain a lithopone of good quality, that would not darken by controlling the zinc oxide

\footnotetext{
${ }^{1}$ Bayer and Company: German Patent, 259,953 (IgII).

2 Brase: German Patent, 254,291 (Sept. 29, 1909).

${ }^{3}$ Ostwald: German Patent, 202,709 (I905).
} 
content, that is, by keeping it down below five percent and above three percent.

The advantage of zinc oxide is that it helps to prevent the yellowing of lithopone.

W. Hurschel ${ }^{1}$ says, "On the other hand, the addition of zinc oxide helps to prevent the yellowing of the oil in a dark, moist room."

It was found by us that the zinc oxide could be replaced by aluminum oxide. It is well known that a soluble aluminum salt in the presence of hydrogen sulphide forms aluminum hydroxide, a gelatinous precipitate. This precipitate is adsorbed by the zinc sulphide. On dehydration, a very inert film of aluminum oxide is obtained.

Grinding lithopone with five percent flowers of sulphur gives a mixture which is a little less sensitive to light. It is probable that a more efficient protection could be obtained by precipitating sulphur in a finely divided state on the surface of the lithopone.

The results of this paper can be summed up as follows:

1. Quenching in water prevents further oxidation of the red hot zinc sulphide. It also disintegrates the semifused mass and dissolves out most of the soluble salts.

2. Heating the barium sulphate-zinc sulphide precipitate is necessary to dehydrate the zinc sulphide and to change its physical condition, so that it forms a dense mass with good body which can be ground more readily.

3. The yellow color produced on over-heating is due to an oxide film as was shown by Farnau.

4. The darkening of lithopone is not due to impurities such as iron, lead, cadmium, etc.

5. The presence of salts which form soluble zinc salts such as sodium chloride, sodium sulphate, etc., accelerates the darkening of the lithopone. These salts dissolve away the zinc oxide film. This is similar to the behavior of magnesium in water. Magnesium does not decompose water very readily

${ }^{1}$ Hurschel: Farben $Z$ tg., I7, 1592-3 (I9I2). 
at ordinary temperatures. In the presence of magnesium chloride, however, the action takes place vigorously.

6. The presence of salts which form insoluble zinc salts such as the alkali phosphates, bicarbonates, ferrocyanides and borates, retards or prevents the darkening of lithopone.

7. The action of light on the zinc sulphide is a reducing one, hydrogen sulphide and metallic zinc being formed.

8. The reaction is not a reversible one, the metallic zinc formed is oxidized to the white zinc oxide.

9. Barium sulphate is not necessary for the darkening of the zinc sulphide.

Io. Heating the zinc sulphide is not necessary to get it to darken, although heating makes the zinc sulphide more sensitive to light, probably because the reducing atmosphere and the sodium chloride used removes the zinc oxide film more efficiently.

II. The zinc oxide film can be removed by boiling in a concentrated solution of zinc chloride. The zinc sulphide so treated will darken in the presence of a reducing agent.

I2. When barium sulphate is precipitated with the zinc sulphide, it aids the darkening due to the fact that it adsorbs the zinc sulphide, thereby giving increased surface exposure of the zinc sulphide. It probably also adsorbs the metallic zinc.

I3. The zinc sulphide will darken without the presence of a reducing agent if it is precipitated with barium sulphate and boiled in a concentrated solution of zinc chloride. The barium sulphate probably adsorbs metallic zinc as well as zinc sulphide, thus making the latter sensitive to light.

I4. The patented processes for the prevention of the darkening of lithopone depend upon the fact that they form an insoluble film around the zinc sulphide.

I5. It is impossible to make a lithopone that will not darken unless there is a film protection of some kind over the zinc sulphide.

I6. We made a lithopone of good quality that would not darken by producing an oxide film on the zinc sulphide and 
keeping the oxide content above 3 percent and below 5 percent. Aluminum oxide can be substituted for zinc oxide. A film of sulphur protects to some extent; no experiments were made to determine the maximum efficiency possible.

In conclusion, the author wishes to acknowledge the kind help of the chemical faculty, especially Dr. Bennett. This research was suggested by Prof. Bancroft and carried out largely under his direction; I wish to express my great appreciation for his kindly advice and criticisms.

Cornell University 